\title{
Isolating Moving Anatomy in Ultrasound Without Anatomical Knowledge: Application to Computer-Assisted Pericardial Punctures
}

\author{
A. Bzostek ${ }^{1}$, G. Ionescu ${ }^{2}$, L. Carrat ${ }^{2}$, C. Barbe ${ }^{2}$, O. Chavanon ${ }^{2,3}$, J. Troccaz ${ }^{2}$ \\ ${ }^{1}$ CIS Laboratory, Department of Computer Science, The Johns Hopkins University \\ ${ }^{2}$ TIMC Laboratory, Faculté de Médecine de Grenoble \\ ${ }^{3}$ Cardiac Surgery Department, Grenoble University Hospital
}

\begin{abstract}
This paper presents an approach to semiautomatic segmentation over a time series without the use of a priori geometric anatomical knowledge, and demonstrates its applicability to pericardial effusion segmentation from ultrasound. The described technique solves the problem in two stages, first automatically calculating a set of exclusion zones, then leveraging the surgeon's anatomical knowledge, simply and interactively, to create a region which corresponds to the stable region within the target effusion. In preliminary testing, the system performs well versus manual segmentation, outperforming it both in terms of perceived quality, as measured in a blinded comparison by an expert, and in terms of time required for generation.
\end{abstract}

\section{Introduction}

Significant work has been devoted to the extraction of anatomical contours in medical imagery from various modalities. Ultrasound imaging poses particular challenges because of its inherently low signal-to-noise ratio, and the dependence of its images on the angle and texture of the target anatomy. Its safety and non-invasiveness, however, maintain this technique's popularity among practitioners. In order to overcome ultrasound's difficulties, most previous attempts at automated segmentation have either relied upon the coding of anatomical knowledge into the recognition procedure, or involved a user heavily in the segmentation process, especially in its initialization. In this paper we present a technique for segmenting moving anatomy which does not rely on any coded knowledge about the target anatomy save that that it is moving cyclically. Instead of in-depth knowledge of the anatomy, the presented algorithm uses knowledge about the ultrasound image formation, and two simple pieces of user input to generate a "safe" segmentation of a pericardial effusion. Because this input is used as the last step of the segmentation process, as opposed to its initialization, the inputs' affects can be viewed and adjusted interactively. By combining simple information over time with this small amount of interactive input, the system is able to achieve a general segmentation of anatomy, even when segmentation of that anatomy within the individual images is quite difficult. Not only is the presented technique nearly automatic, but it produces segmentations which appear to be at least as good as those produced by expert humans, in less time as well. This paper will first present a short overview of previous, related work and an outline of the target procedure. After presenting the technique, we will then 
present the results from preliminary validation experiments, and will conclude with possible directions for further work.

\section{Background}

Pericardial effusions are created when, due to disease or injury, fluid collects between the heart and the pericardium, an outer, sack-like sheath that surrounds it. It is particularly common after cardiac surgery [1]. In order to avoid complications, including cardiac tamponade and constrictive pericarditis, the fluid must be drained. This can be achieved through access established percutaneously, using a needle under ultrasound guidance. The minimally invasive puncture poses many difficulties to the surgeon relating both to the difficulty of the task and the extreme sensitivity to errors, particularly in overinsertions, where the epicardium can be punctured. Consequently, surgeons are often reticent to approach small $(<\sim 15 \mathrm{~mm})$ or badly placed effusions percutaneously and thus open surgery is preferred in these cases.

\subsection{Computer-Assisted Pericardial Puncture}

In order to assist surgeons in this task, a system, CASPER, was developed by Barbe, et al $[2,3]$ which provides computer-based guidance during the puncture. They have shown that there exists, for these effusions, a stable target area which remains within the effusion throughout the cardiac cycle. Using CASPER, a surgeon defines this "safe" target area for the needle insertion based on ultrasound imagery registered using an Optotrak IR tracking system. This is accomplished by first, allowing the user to segment the effusion in each of a set of nearly parallel images, then, combining these segmentations to generate the minimal common target area. Again using the IR tracker, the system follows the puncture needle and provides feedback to the surgeon. The feedback combines this positional information with the "safe" target and an insertion trajectory defined intraoperatively. This process was satisfactory for validation, but is labor intensive and needs to be more automated to allow the use of the system clinically. The work presented in this paper attempts to automate this segmentation process.

\subsection{Related Work}

Automation of image-based medical tasks has been a target of research for some time. Consequently, there is a large body of work dedicated to this sometimes difficult task. Potentially applicable groundwork has been done in texture analysis [4-12] and optical flow [13-19].

Quite a bit of work has been particularly focused on the segmentation of the beating heart from time-sequences of ultrasound images. Though the modality and target anatomy of these systems are the same, their objective and restrictions are different from ours. Previous work has focused primarily on the segmentation of the left ventricle (LV) for geometric and functional analysis [20-28]. Many of these techniques [21-23,25,27] rely on user input for initialization information, and all of them rely on the fact that the image of the target (LV) has some known geometric properties. Additionally, none of these technique are intraoperative, and thus do not have tight time constraints. While our required segmentation is simpler than those used for LV analysis, the lack of known 
view orientation and target geometry, as well as the time requirements imposed intraoperatively make the problem an interesting one.

\section{Methods}

The method we have developed is divided into two stages. First, a set of "safe" regions are generated automatically. Using only our knowledge that the target anatomy (heart and pericardium) will either be moving cyclically, or stable and give high response, these regions are created as to not contain any such anatomy in any of the used images. Second, using three pieces of user input, a target zone is calculated which stays within the "safe" regions. Because the first component is computed completely automatically, interactive manipulation of the input and viewing of the resulting target area is possible.

\subsection{Image Collection}

Images are from a PAL video stream generated by the ultrasound imager. When using a curved probe, imager calibration techniques described in [3] are used to calbrate the imager and covert the image into its inherant polar coordinates.

\subsection{Safe Region Generation}

The generation of anatomy-free regions is accomplished in three steps:

- Segmentation: A rough segmentation, which includes the target anatomy, is generated for each image.

- Selection: A set of pixels, or seeds, which touch regions of target anatomy are selected for the set of images.

- Summation: Portions of the segmentations which are touched by seeds are summed to generate "unsafe" areas which contain target anatomy.

Segmentation. Each rough segmentation is generated using a non-isotropic Laplacian of Deriche (LoD) filter [28], applying a high-pass horizontal run-length filter, and then removing very small groups.

Ultrasound image response falls off dramatically as the angle between the measurement wave and the imaged surface decreases below $45^{\circ}$. Thus, only anatomy whose surface is near perpendicular to the probe direction will be well imaged. Systematic image components (connected groups of high response), which run vertically can thus be considered noise. By applying a non-isotropic LoD, with a small $\alpha$ (large standard deviation, $\beta$ of the corresponding Gaussian Curve) in the horizontal direction, and large $\alpha$ vertically, the width of near vertical image components is highly reduced. Then, using a horizontal run-length filter, narrow, vertical segmented regions are removed.

Finally, the remaining groups with less than a low threshold size are removed. The resulting segmentation removes much of the systematic noise inherent in the ultrasound images (see example 1). This segmentation captures the target anatomy to a very high degree. Unfortunately, it also segments other components in the image. 


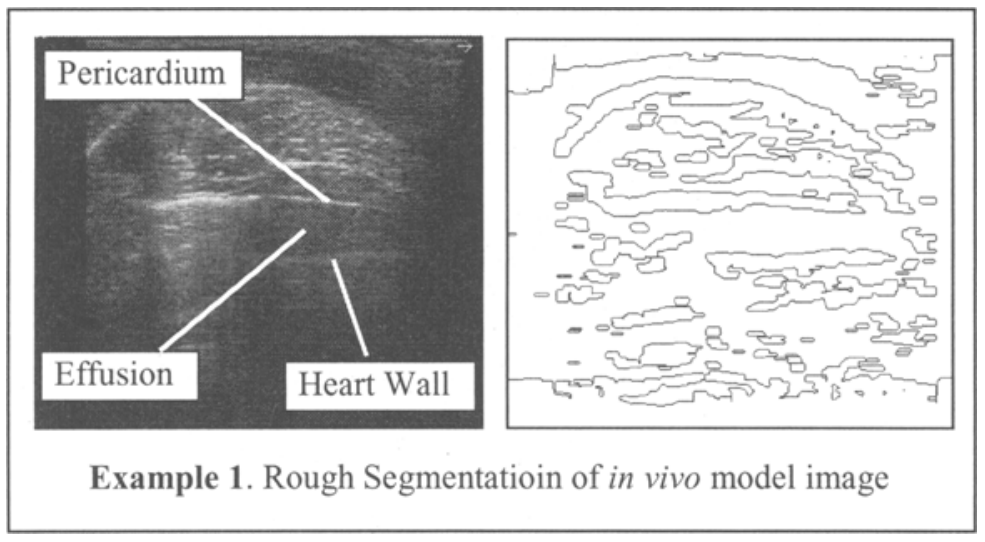

Selection In order to find important areas in the image, sets of seed pixels are selected, based on sets of attributes calculated per pixel. Two sets of seeds are generated, one corresponding to static, one to moving anatomy. Together, these seed pixels touch both the parts of the image which are strong and stable (pericardium), and those whose strength varies cyclically (heart).

The static seeds are generated simply by calculating the energy (sum squared of activation) at each pixel over all images, and thresholding these values so that a target percentage of the image is covered.

The dynamic seeds are calculated using a similar procedure, except instead of measuring a single attribute, each pixel's value is calculated as the product for four separate, but related measures:

- $\quad \mathrm{S} / \mathrm{N}$ Ratio of maximum Harmonic

- Power of maximum Harmonic

- Log of the size of the pixel's uniform max harmonic frequency group

- Edge "Pass Over" Count

These attributes measure, respectively, how strongly a pixel's response over time corresponds to a cycle, how strong that cycle is, how large of a connected group is moving in the same cycle, and how much segmented edge motion passes over the pixel. Pixels with high values in all of these attributes are highly likely to lie in regions of the image which contain cyclically moving anatomy, though these pixels will not cover all such regions (see example 2).

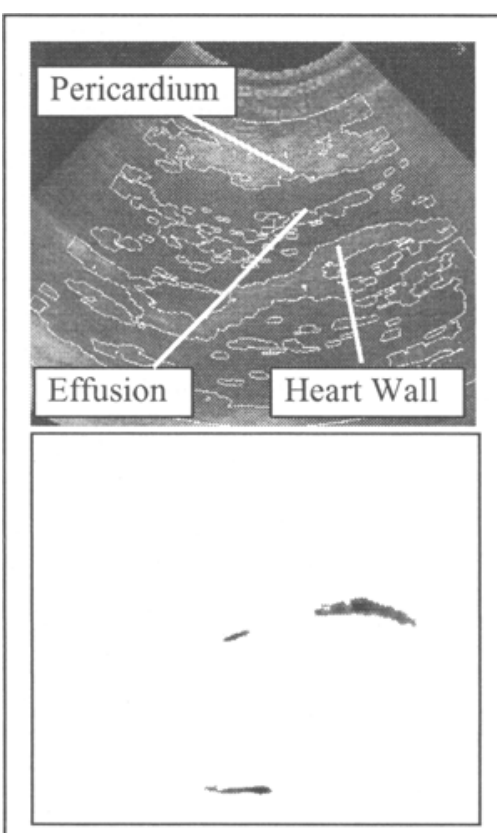

Example 2. Human Images: TOP: Image w/ rough segmentation, BOTTOM: Dynamic Seeds from the same image set (corrected for curvature) 
Summation Once the seeds are determined, we use these, in conjunction with the segmented regions, to generate areas which could contain target anatomy. For each image, a set of pixels is marked which, moving only through regions marked as "inside" by the segmentation, are within a threshold distance of a seed pixel, and which belong to connected marked groups larger than a small threshold size. Any pixel that is marked for any image is considered "unsafe". Thus, those pixels not marked in any image comprise the "safe" zones (see example 3).

\subsection{Target Area Selection}

The automatically generated "safe" zones now constrain the choice of a target area, but in order to actually select a target from the image, anatomical knowledge is necessary. By allowing the user to select a target area in a simple way, we leverage the surgeon's anatomical knowledge in a non-obtrusive manner.

\section{User Input}

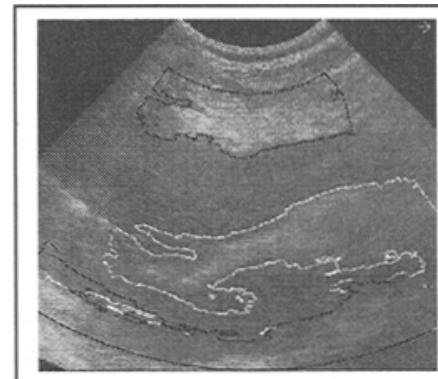

Example 3. Human Image w/ contours of "Unsafe" regions Black contoursgenerated from static seeds, white regions- generated from dynamic ones

User input consists of three things: a center point, a maximum radius, and a smoothing value for the target area. Because the creation of the "safe" zones from the summation process need not be repeated, the values specifying the target area can be inputted and modified interactively until the user is satisfied.

\section{Area Generation}

Given the user input, a target zone is generated by marking all pixels within the safe zone within the inputted radius from the center, as calculated by traversing only pixels in this zone (similar to the procedure for the seeding of the segmentations in the previous section). An opening of the specified size is then applied and the unconstrained, rounded ends are removed. (see Example 4).

\section{Results}

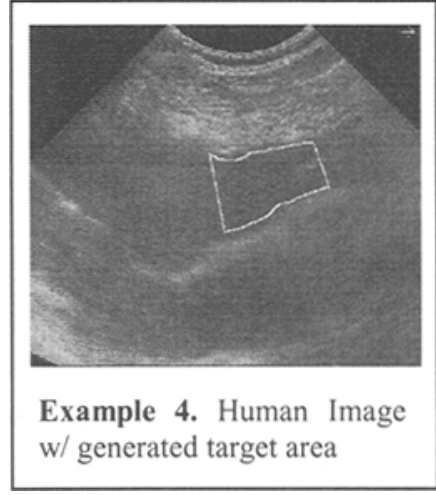

In order to validate this technique and compare it to manual segmentation, three approaches have been used to date. First, manual segmentations of recorded image sets have been compared directly to segmentations generated automatically (using the center point and radius from the manually generated regions instead of user input). Second, using these same segmentations, a human expert has been asked to rate and compare the segmentations without knowing how they were generated. Third, in order to compare times, automated segmentations were generated on the intra-operative system, using prerecorded data. 


\subsection{Difference Comparison}

Using the center point and maximum radius from manually generated segmentations, automatic segmentations were generated for 10 recorded data sets. The parts of the segmentations corresponding to the inner and outer walls of the effusion were then compared. A difference measure was generated by filling the area between the contours (inner-to-inner, outer-to-outer), then dividing the area of these regions by their length. The differences averaged $3.5 \mathrm{~mm}$, with a maximum difference of $5.0 \mathrm{~mm}$.

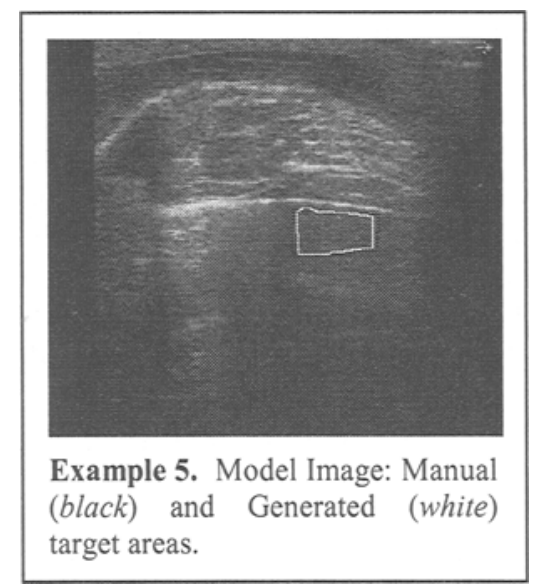

\subsection{Blinded Survey}

The differences found in the last test cannot necessarily be taken as errors on the part of the automatic procedure. It is possible that they fall within the range of acceptable segmentations. To test the quality of the generated segmentations, an expert human (a cardiac surgeon), was asked to compare the segmentations (both automatic and manual) without knowledge as to how the segmentations were generated. As rated by this surgeon, the automatic segmentations were favored overall, and individually in 8 of 10 cases. In the two cases in which the manual segmentation was rated as better, the surgeon rated the automated segmentation as "too conservative".

\subsection{Comparison in Intra-Operative System}

Running on the same system, the time required for the semi-automatic segmentation was compared against that for the manual segmentation. The semi-automated system requiried approximately 5 minutes ( 4 minutes of automatic processing, 1 minute of manual selection), while the purely solution required 10 .

\section{Future Work}

More work needs to be done in order to validate the proposed system. Investigations need to be conducted in an in-vivo environment and more extensive work needs to be conducted on human effusion images. We are very encouraged by the current results and do not foresee any significant obstacles.

Additionally, there are many ways to extend this work, some of which are being actively investigated. The choice of selection measures is heuristic and thus open to significant extension. Work is currently being done to assess time-texture measures, particularly simple measures such as entropy, as components for this measure.

The automated component of the system could be applied as an initial processing step for a more complete segmentation, allowing a region of interest and geometry approximation to be constructed before a detailed image-by-image segmentation is conducted. The complete system could also be applicable to other procedures where segmentation of each of a series of images is difficult, but where a composite segmentation is the objective. 


\section{Conclusions}

We have presented an approach to semi-automatic segmentation which seeks not to capture the anatomical knowledge of a surgeon, but to utilize it efficiently. As applied to pericardial effusion segmentation, it seeks to generate a composite segmentation from a series of images none of which can be well segmented individually. Rather than an initialization, user interaction is utilized as a final step allowing the interactive manipulation of the inputs and the watching of their affects on the final result. In the preliminary testing so far conducted, the system has performed well and takes less time than manual segmentation. And, while its segmentations sometimes differ from those generated manually by small amounts, it appears that those it generates are not only adequate, but usually better than those created by hand, and, in those cases where they are worse, the segmentations are more conservative. Combining this with the CASPER system's already demonstrated guidance capability could give surgeons the confidence to approach a variety of effusions percutaneously.

\section{Acknowledgements}

Supported by NSF Whitaker Foundation Program on Cost-Reducing Health Care Technologies (grant \# BES9520501) and INSERM PSTRC grant (GMCAO project \# PARMPSTRC9607).

\section{References}

1. J. Sahni, T. Ivert, I Harzfeld and L. Bodin. "Late cardiac Tamponade after Open-Heart Surgery," Scan J. Thorac Cardiovasc Surg 25, pp. 63-68, 1991.

2. Barbe, C., Carrat L., Chavanon O., and Troccaz, J., "Computer Assisted Pericardic Surgery," Computer Assisted Radiology, pp. 781-786, 1996.

3. Chavanon, O., Barbe, C., Troccaz, J., Carrat, L., Ribout C., Blin, D.," Computer Assisted PERicarial punctures: animal freasability study," Proc. CVRMed-MRCAS '97, pp. 285-294, 1997.

4. Mir, A.H.; Hanmandlu, M.; and Tandon, S.N.; "Texture analysis of CT images"; IEEE Engineering in Medicine and Biology; 781-786; December 1985

5. Bleck, J.S.; Ranft, U.; Hecker, H.; Bleck, M.W.; Thiesemann, C.; Wagner, S.; and Manns, M.; "Random field models in textural analysis on ultrasonic images of the liver" IEEE Trans. Medical Imaging, vol. 15, no. 6, pp. 796-801, Dec. 1996.

6. Conners, R.W.; and Harlow, C.A.; "A theoretical comparison of texture algorithms"; IEEE Trans. Pattern Analysis and Machine Intelligence; Vol. PAMI-2; No. 3; pp. 204-222; May 1980.

7. Hall, E.L.; Crawford, W.O.; and Roberts, F.E.; "Computer classification of pneumoconiosis from radiographs of coal workers"; IEEE Trans. Biomedical Engineering; vol. BME-2; No. $1 ;$ pp. 518-52; Nov 1985.

8. Lizzi, F.L.; "Relationship of ultrtasonic spectral parameters to features of tissue microstructure" IEEE Trans. Sonics and Ultrasonics, vol. UFFC-33, no. 3, pp. 319-329, May 1986.

9. Sutton, R.; and Hall, E.L.; "Texture measures for automatic classification of pulmonary diseases; IEEE Trans. on Computers; Vol. C-2; No7; pp667-676; July 1972.

10. Tsatsanis, M.K.; and Giannakis, G.B.;, "Object and texture classification using higher order statistics" IEEE Trans. Pattern Analysis and Machine Intelligence, vol. 14, no. 7, pp. 733749 , July 1992. 
11. Wu, C.M.; Chen, Y.c.; and Hsieh, K.S., "Texture features for classification of ultrasonic liver images", IEEE Trans. Medical Imaging; vol 11; no. 2; pp. 141-152; June 1992.

12. Kruger, R.P.; Thompson, W.B.; and Turner, A.F.; "Computer diagnosis of Pneumoconiosis"; IEEE Trans. Systems, Man and Cybernetics; vol. SMC-4; no 1 ;pp 40-49; Jan .1974.

13. Meunier, J.; Sehboub, Z.; Bertrand, M.; and Lesperence, J.; "Estimation of the left ventricle 3-D motion from single plane cineangiograms"; Not. $A v .1993$

14. Mailloux, G.E.; Bleau, A.; Bertrand, M.; and Petitclerc, R.; "Computer analysys of heart motion from two-dimensional echocardiograms"; IEEE Trans. Medical Imaging; vol BME34; no4; pp356-364; May 1987.

15. Mailloux, G.E.; Langlois, F.; Simard, P.Y.; and Bertrand, M.; "Restauration of the velocity field of the heart from two-dimensional echocardiograms"; IEEE Trans. Medical Imaging; vol8 no 2, pp. 143-153; June 1989.

16. Thomson, W.B.; Mutch, K.M.; and Berzins, V.A.; "Dynamic occlusion analysis on optical flow fields"; IEEE Trans. Pattern Analysis and Machine Intelligence; PAMI-7; no 4. pp 373383, July 1985.

17. Meunier, J,; and Bertrand, M.;, "Caracterisation des tissus par analyse de mouvement de la granularite echographique" Innov. Tech. Biol. Med., vol. 15, no. 3, pp. 268-280, 1994.

18. Meunier, J.; and Bertrand, M.;, "Ultrasonic texture motion analysis : theory and simulation" IEEE Trans. Medical Imaging, vol. 14, no. 2, pp. 293-300, June 1995.

19. Schunk, B.G.;, "Image flow segmentation and estimation by constraint line clustering" IEEE Trans. Pattern Analysis and Machine Intelligence, vol. 11, no. 10, pp. 1010-1027, Oct. 1989.

20. Han, C.Y., Kwun, N.L., Wee, W.G., Mintz, R.M., Porembka, D.T., "Knowledge-Based Image Analysis for Automated Boundary Extraction of Transesophogeal Echocardiographic Left-Ventricular Images," IEEE Transactions on Medical Imaging, vol. 10, No. 4, pp. 602610, December 1991.

21. Kingler, J. W., Vaughn, C. L., Fraker, T. D. Andrews, L. T., "Segmentation of Echocardiographic Images Using Methematical Morphology," IEEE Transactions on Biomedical Engineering, vol. 35, no. 11, pp. 925 - 934, November, 1988.

22. Chu, C. H., Delp, E. J., Buda, A. J., "Detecting Left Ventricular Endocardial and Epicardial Boundaries by Digital Two-Dimensional Echocardiography", IEEE Trans. On Medical Imaging, vol. 7, no. 2, June 1988.

23. Teles de Figueiredo, M., Leitão, J. M. N., "Baysian Estimation of Ventricular Contours in Angiographic Images", IEEE Transactions on Medical Imaging, vol. 11, no. 3, September 1992.

24. Dias, J. M. B., Leitão, J. M. N., "Wall Position and Thickness Estimation from Sequences of Echocardiographic Images," IEEE Trans. On Medical Imaging, vol. 15, no. 1, February 1996.

25. Lilly, P, Jenkins, J., Bourdillon, P., "Automatic Contour Definition on Left Ventriculograms by Image Evidence and a Multiple Template-Based Model," IEEE Trans. On Medical Imaging. Vol. 8, no. 2, June 1989.

26. Friedland, N, Adam, D., "Automatic Ventricular Cavity Boundary Detection from Sequention Ultrasound Images Using Simulated Annealing," IEEE Trans. On Medical Imaging, vol. 8, no. 4, December 1989.

27. Detmer, Paul R., Bashein, G., Martin, R. W., "Matched Filter Identification of LeftVentricular Endocardial Borders in Transesophageal Echocardiograms," IEEE Trans. On Medical Imaging, vol. 9, no. 4, December 1990.

28. Deriche, R, "Fast Algorithms for Low-Level Vision", IEEE Transactions of Pattern Analysis and Machine Intelligence, vol. 12, no. 1, January 1990.

29. Press, W. H., Teukolsky, S. A., Vetterling, W. T., Numerical Recipes in C: The Art of Scientific Computing, $2^{\text {nd }}$ Edition, Cambridge Univ. Pr., January 1993. 